\title{
Influence of side chain structure on the thermal and antimicrobial properties of cationic methacrylic polymers
}

\author{
Rocío Cuervo-Rodríguez ${ }^{1}$, Alexandra Muñoz-Bonilla ${ }^{2,3^{*}}$, Javier Araujo ${ }^{2}$, Coro Echeverría ${ }^{2,3}$ and \\ Marta Fernández-García ${ }^{2,3^{*}}$ \\ ${ }^{1}$ Facultad de Ciencias Químicas (UCM), Av. Complutense s/n, Ciudad Universitaria, 28040 Madrid, Spain \\ ${ }^{2}$ Instituto de Ciencia y Tecnología de Polímeros (ICTP-CSIC), C/Juan de la Cierva 3, 28006 Madrid, Spain \\ ${ }^{3}$ Interdisciplinary Platform for Sustainable Plastics towards a Circular Economy, SUSPLAST \\ E-mail: martafg@ictp.csic.es (M.F-G.), sbonilla@ictp.csic.es (A.M-B.)
}

\begin{abstract}
A novel methacrylic monomer, 2-(((2-(4-methylthiazol-5-yl)ethoxy)carbonyl)oxy)ethyl methacrylate (MTZ), containing in the lateral chain both hydrolytically sensitive carbonate and thiazole groups has been firstly described. Posterior, it was free-radical polymerized and copolymerized with acrylonitrile in anhydrous solution of dimethylsulfoxide, with the purpose of investigate the influence of its chemical and structural characteristics on the antimicrobial activity. Cationic antimicrobial polymers were subsequently obtained by alkylation reaction with methyl and butyl iodide agents. Their thermal behavior was analyzed in all series, modified and unmodified copolymers in terms of glass transition temperature and thermal stability. The antimicrobial activity in solution was tested against Gram-positive Staphylococcus aureus and Staphylococcus epidermidis, Gram-negative Escherichia coli and Pseudomonas aeruginosa bacteria, and Candida parapsilosis yeast and further correlated with their zeta potential. Then, blends of these cationic polymers with commercial polyacrylonitrile as matrix material were prepared and their effectiveness as antimicrobial coating was determined against Gram-negative Escherichia coli bacteria. In addition and due to the presence of the carbonate groups in the copolymer structures, these systems could be further used as antimicrobial releasing coatings in alkaline conditions.
\end{abstract}

Keywords: Cationic copolymers; thiazole; stability; charge density; thermal; blends; antimicrobial

\section{Introduction}

The rising prevalence of drug-resistant pathogenic microorganisms together with the dwindling supply of new antibiotics is one on the most serious global health concerns in modern medicine $[1,2]$. This context has encouraged scientist to research for new antimicrobial agents as an alternative to traditional antibiotics. In the last years, cationic polymers have aroused considerable attention as antimicrobial agents due to their outstanding properties. Compared to small cationic compounds, polymers such as those based on quaternary ammonium, phosphonium, imidazolium, and pyrrolidinium groups exhibit higher antimicrobial activity, chemical stability, lower toxicity and low propensity to induce resistance [3-6]. Unlike traditional antibiotics, the mechanism of action against bacteria is mainly by membrane disrupting, which is assumed to be the reason of this low susceptibility to generate resistance, as this pathway is more difficult for bacteria to circumvent. Although the mode of action is not completely understood, the mechanism seems to involve adsorption of the polymers onto the negatively charged bacterial membrane through electrostatic interactions, disruption of the wall also by penetration and insertion of hydrophobic segments into the inner part of the membrane, and cell lysis and release of cytoplasmic components, which finally leads to bacterial death [7]. Typically, Gram-negative bacteria with an additional outer membrane result less susceptible to cationic polymers than Gram-positive with a single membrane [8]. Most of the bacterial membrane are negatively charged while mammalian cells have a net neutral charge on the surfaces provided by zwitterionic phospholipids, fact gives selectivity and then low cytotoxicity [9]. Therefore, 
from this mechanism it is clear that in addition to positive charge, the antimicrobial polymers should have hydrophobic segments, i.e. an appropriate hydrophilic/hydrophobic balance, to facilitate their permeation through the apolar lipid bilayers of the bacteria wall. Indeed, host defense antimicrobial peptides (HD-AMP) with amphiphilic structure have been a source of inspiration to design and develop new synthetic antimicrobial peptides and polymers [10-13]. Conventional synthetic polymers including amphiphilic polymethacrylates, polystyrenes or poly(vinyl pyridine)s mimicking AMP, offer many advantages over synthetic peptides made from artificial amino acids, such as stability and versatility, but principally their synthesis allows cost effective large scale production. Synthetic polymers can be easily synthesized from a huge range of monomers of very different functionalities, and their composition, architecture and molecular weight can be finely tuned using controlled polymerization techniques $[14,15]$. Our group [16-18] has developed several cationic polymethacrylic systems bearing thiazolium group as side chain by widely employed polymerization techniques such as conventional and controlled radical polymerizations. Compared to polymers bearing quaternary ammonium groups, these polymers based on thiazolium groups have been less explored, but it was demonstrated their excellent antimicrobial properties against a broad spectrum of microorganisms. In those publications, we have systematically studied the influence of polymer chemical structure on the antimicrobial activity, including parameters such as optimal chemical structure and factors affecting the antimicrobial activity of these cationic polymers such as the hydrophobic/hydrophilic balance, the charge density, length of the alkyl chain and molecular weight, among others. In this article, we present a novel monomeric structure (MTZ) bearing thiazole moieties susceptible of quaternization to obtain cationic charge and hydrolytically sensitive carbonate ester moieties, which could be attractive for delivery systems [19]. This new monomer was homo and copolymerized with acrylonitrile (AN) by conventional radical polymerization in order to obtain the corresponing amphiphilic antimicrobial polymers. The resulting cationic polymers obtained after $\mathrm{N}$-alkylating reaction were chemical and thermal characterized and the activity against different microorganisms was also analyzed as a function of the hydrophobic/hydrophilic balance, thereby MTZ/AN ratio. Furthermore, antimicrobial coatings were prepared from these cationic amphiphilic copolymers by blending with commercially available polyacrylonitrile (PAN). PAN was chosen as matrix material because presents good thermal, chemical and photo-stability as well as mechanical properties, and is widely used in biomedical applications. Besides, the design to the monomer MTZ includes a carbonate ester group in the side chain, which is hydrolytically sensitive under alkaline conditions and would allow the delivery of cationic moieties, making these systems potential candidate as antimicrobial releasing coatings.

\section{Materials and Methods}

\subsection{Materials}

2-Hydroxyethyl methacrylate (99\%), p-nitrophenyl chloroformate (96\%), 5-(2-hydroxyethyl)-4-methylthiazole (98\%), hydroquinone (99\%), 4-dimethylaminopyridine (DMAP, 99\%), anhydrous tetrahydrofuran (THF, 99.9\%), anhydrous dimethylsulfoxide (DMSO, 99.9\%), anhydrous $\mathrm{N}, \mathrm{N}$-dimethylformamide (DMF, 99.8\%), iodomethane $(99.5 \%)$ and 1 -iodobutane $(99 \%)$ were purchased from Sigma-Aldrich and used as received. Triethylamine, (TEA, 99\%, Sigma-Aldrich) was distilled from potassium hydroxide under inert atmosphere prior to use. Acrylonitrile (AN, 99\%, Sigma-Aldrich) was distilled prior to use. 2,2'-azobisisobutyronitrile (AIBN, 98\%, Acros) was recrystallized twice from methanol. All the organic solvents were of AR grade, dichloromethane (DCM, 99.7\%, Cor Química S.L.), ethyl acetate (EtOAc, 99.8\%, Cor Química S.L.), n-hexane (96\%, Scharlau) were used without further purification. Polyacrylonitrile (PAN, molecular weight of $150 \mathrm{kDa}$ ) was supplied by Sigma Aldrich and was used without previous purification. Cellulose dialysis membranes (CelluSep T-series and $\mathrm{H} 1$ ) were obtained from Membrane Filtration Products, Inc. Round glass coverslips of $12 \mathrm{~mm}$ diameter, obtained from Ted Pella Inc. (Redding, CA, USA). 


\subsection{General Methods and Instrumentation}

Reactions were monitored by thin-layer chromatography (TLC) using $0.50 \mathrm{~mm}$ Merck silica gel F254 TLC glass plates and visualized by UV irradiation. Flash column chromatography was performed using Merck silica gel $60 \AA$ (230-400 mesh). Polyelectrolytes purification was performed by dialysis using cellulose membranes with a molecular weight cut off of $3500 \mathrm{Da}$ that were obtained from Orange Scientific. Nuclear Magnetic Resonance (NMR) spectra were recorded on a Bruker (400 $\mathrm{MHz}$ ) spectrometer at room temperature. Chemical shifts $(\delta)$ are reported in ppm with the residual solvent signal as internal standard (chloroform at 7.26 and $77.00 \mathrm{ppm}$ and dimethylsulfoxide at 2.51 and $39.52 \mathrm{ppm}$ for ${ }^{1} \mathrm{H}$ and ${ }^{13} \mathrm{C}$-NMR spectroscopy, respectively). The data was reported as: $\mathrm{s}$ =singlet, $\mathrm{t}=$ triplet, $\mathrm{m}=$ multiplet or unresolved, $\mathrm{bs}=$ broad singlet, $\mathrm{C}$ quat. = quaternary carbon atom; integration; coupling constant in $\mathrm{Hz}$; assignment. Mass spectrometry (MS) analyses was performed as high resolution ESI measurements on an Agilent 6500 Series Accurate-Mass Q-TOF LC/MS System with the Agilent 1200 LC. Fourier-transform infrared (FTIR) spectra were recorded on a Bruker Alpha instrument with Universal Attenuated Total Reflectance (ATR) Sampling Accessory module and absorptions were given in wavenumbers $\left(\mathrm{cm}^{-1}\right)$. The number-average molecular weight $\left(M_{n}\right)$, weight-average molecular weight $\left(M_{w}\right)$ and polydispersity index (PDI) of polymers were measured by Size Exclusion Chromatography (SEC) with a chromatographic system (Waters Division Millipore) equipped with a Waters model 410 refractive-index detector. $\mathrm{N}, \mathrm{N}$-dimethylformamide (Scharlau, 99.9\%) containing $0.1 \%$ of $\mathrm{LiBr}$ (Aldrich, 99.99\%), was used as the eluent at a flow rate of $1 \mathrm{~mL} / \mathrm{min}$ at $50^{\circ} \mathrm{C}$. Styragel packed columns (HR2, HR3, and HR4, Waters Division Millipore) were used. Poly(methyl methacrylate) standards (Polymer Laboratories, Laboratories, Ltd.) ranging from $2.4 \times 10^{6}$ to $9.7 \times 10^{2} \mathrm{~g} / \mathrm{mol}$ were used to calibrate the columns. The zeta potential values of copolymers was analyzed using the Zetasizer Nano series ZS (Malvern Instrument Ltd., U.K.). The data are the average at least of 5 runs. Glass transition temperatures $\left(T_{g}\right)$ of all copolymers were measured by differential scanning calorimetry (DSC) using a TA Q2000 instrument equipped with an intercooler for low temperatures. The samples were equilibrated at -70 ${ }^{\circ} \mathrm{C}$ and heated to $150^{\circ} \mathrm{C}$, then cooled to $-70^{\circ} \mathrm{C}$ and again heated to $150^{\circ} \mathrm{C}$ in all the runs at $10^{\circ} \mathrm{C} / \mathrm{min}$ under dry nitrogen $\left(50 \mathrm{~cm}^{3} / \mathrm{min}\right)$.

\subsection{General Biological Instrumentation and Microorganisms}

$\mathrm{NaCl}$ solution $(0.9 \%$, BioXtra, suitable for cell culture), DMSO (BioReagent, for molecular biology, suitable for plant cell culture, $\geq 99.9 \%$ ), phosphate buffered saline powder (PBS, pH 7.4) and Triton $^{\mathrm{TM}} \mathrm{X}$-100 solution (BioUltra, for molecular biology, 10\% in $\mathrm{H}_{2} \mathrm{O}$ ) were purchased from Aldrich and were used as received. In vitro antimicrobial tests were performed in 96-well microplates (BD Biosciences) using a Finnpipette ${ }^{\mathrm{TM}} \mathrm{F} 2$ (Thermo Scientific) as a multichannel pipette and appropriately sterilized sized pipette tips. Sheep blood (5\%) Columbia Agar plates were purchased from bioMérieux and $\mathrm{BBL}^{\mathrm{TM}}$ Mueller Hinton broth was purchased from Becton, Dickinson and Company and was used as a microbial growth media. American Type Culture Collection (ATCC): Gram-positive Staphylococcus aureus (S. aureus, ATCC $\circledast$ 29213), Staphylococcus epidermidis (S. epidermidis, ATCC $\AA$ 12228), and Gram-negative Escherichia coli (E. coli, ATCC $\AA^{2}$ 25922), Pseudomonas aeruginosa ( $P$. aeruginosa, ATCC $\AA$ 27853) bacteria and Candida parapsilosis (C. parapsilosis, ATCC $\circledast 22109$ ) yeast were purchased from Oxoid ${ }^{\mathrm{TM}}$. Microorganisms were incubated at $37^{\circ} \mathrm{C}$ in a Jouan IQ050 incubator. The optical density of the microorganism suspensions was measured in McFarland units proportional to microorganism concentration by a DensiCHEK ${ }^{\text {TM }}$ Plus (VITEK®, bioMérieux).

\subsection{Experimental procedures}

2.4.1. Synthesis of 2-((2-(4-methylthiazol-5-yl)ethoxy)carbonyl)oxy)ethyl methacrylate monomer, MTZ

The activated HEMA monomer with $p$-nitrophenyl chloroformate, HEMAN, was prepared according to the procedure described previously [20]. Then, in an oven-dried $500 \mathrm{~mL}$ three-necked 
round-bottomed flask containing a magnetic stirring bar, HEMAN $(4.00 \mathrm{~g}, 13.6 \mathrm{mmol})$ and hydroquinone $(0.16 \mathrm{~g})$, to avoid the possible polymerization reaction, were dissolved in anhydrous THF $(80 \mathrm{~mL})$ at room temperature under argon atmosphere. Subsequently, in anhydrous THF (20 $\mathrm{mL}$ ) 5-(2-hydroxyethyl)-4-methylthiazole $(2.134 \mathrm{~g}, 15 \mathrm{mmol})$ was successively added. After addition of freshly distilled TEA $(4 \mathrm{~mL})$, the reaction was performed at $70^{\circ} \mathrm{C}$ for $24 \mathrm{~h}$ while stirring under inert atmosphere (see Scheme 1). The reaction mixture was cooled down up to room temperature and concentrated under reduced pressure to afford dark orange oil. This mixture was purified to remove byproducts ( $p$-nitrophenol and trimethylamine chlorohydrate) and unreacted reagents by flash column chromatography (silica gel; DCM and DCM/EtOAc 9:1) to give MTZ as clear yellow oil (3.25 g, $80 \%$ yield).<smiles>C=C(C)C(=O)OCCO</smiles>

HEMA<smiles>C=C(C)C(=O)OCCOC(=O)OCCc1scnc1C</smiles>

MTZ<smiles>C=C(C)C(=O)OCCOC(=O)Oc1ccc([N+](=O)[O-])cc1</smiles><smiles>Cc1ncsc1CCO</smiles>

Scheme 1. Synthesis of MTZ monomer.

${ }^{1} \mathrm{H}-\mathrm{NMR}\left(300 \mathrm{MHz}, \mathrm{CDCl}_{3}\right) \delta(\mathrm{ppm}): 8.58(\mathrm{~s}, 1 \mathrm{H},=\mathrm{CH}$ thiazole $), 6.12(\mathrm{~s}, 1 \mathrm{H},=\mathrm{CH}), 5.58(\mathrm{~s}, 1 \mathrm{H}$, $=\mathrm{CH}), 4.36\left(\mathrm{bs}, 4 \mathrm{H}, 2 \mathrm{COOCH}_{2} \mathrm{CH}_{2} \mathrm{OCOO}\right), 4.29\left(\mathrm{t}, 2 \mathrm{H}, \mathrm{J}=6.0 \mathrm{~Hz}, \mathrm{OCH}_{2}\right), 3.13(\mathrm{t}, 2 \mathrm{H}, \mathrm{J}=6.0 \mathrm{~Hz}$, $\mathrm{CH}_{2}$ ), 2.39 (s, 3H, $\mathrm{CH}_{3}$ thiazole), $1.92\left(\mathrm{~s}, 3 \mathrm{H}, \mathrm{CH}_{3}\right) \cdot{ }^{13} \mathrm{C}-\mathrm{NMR}\left(75 \mathrm{MHz}, \mathrm{CDCl}_{3}\right) \delta$ (ppm): 167.04 (C-4), 154.77 (C-7), 150.20 (C-12), 150.04 (C-11), 135.79 (C-3), 126.26 (C-1), 125.96 (C-10), 67.50 (C-5), 65.71 (C-6), 62.11 (C-8), 25.78 (C-9), 18.26 (C-13), 14.86 (C-2). FTIR (neat): 2961, 1745, 1716, 1637, 1546, 1254, $1155 \mathrm{~cm}^{-1}$; HR-MS (ESI): $\mathrm{m} / \mathrm{z}$ required for $\mathrm{C}_{13} \mathrm{H}_{18} \mathrm{NO}_{5} \mathrm{~S}\left[\mathrm{M}^{+} \mathrm{H}\right]^{+}, 300.09002$; found, 300.08986 .

\subsubsection{Synthesis of copolymers, $P(A N-c o-M T Z)$}

A series of $\mathrm{P}(\mathrm{AN}-\mathrm{co}-\mathrm{MTZ}) \mathrm{s}$ copolymers were synthesized via conventional radical polymerization of acrylonitrile and MTZ using different feed ratio as shown in Scheme 2. Briefly, in a Schlenk tube, both comonomers were added in a total concentration of $1 \mathrm{M}$ in anhydrous DMSO and with a concentration of the initiator, AIBN, of $5 \times 10^{-2} \mathrm{M}$. After purging the mixture with argon, the solution was stirred at $60^{\circ} \mathrm{C}$ for $20 \mathrm{~h}$. After this period, the mixture was dialyzed against distilled water with the exception of polyacrylonitrile, which was precipitated in distilled water under vigorous stirring and then collected by filtration and washed several times with water. The solid was dried under vacuum for $24 \mathrm{~h}$ until constant weight. 

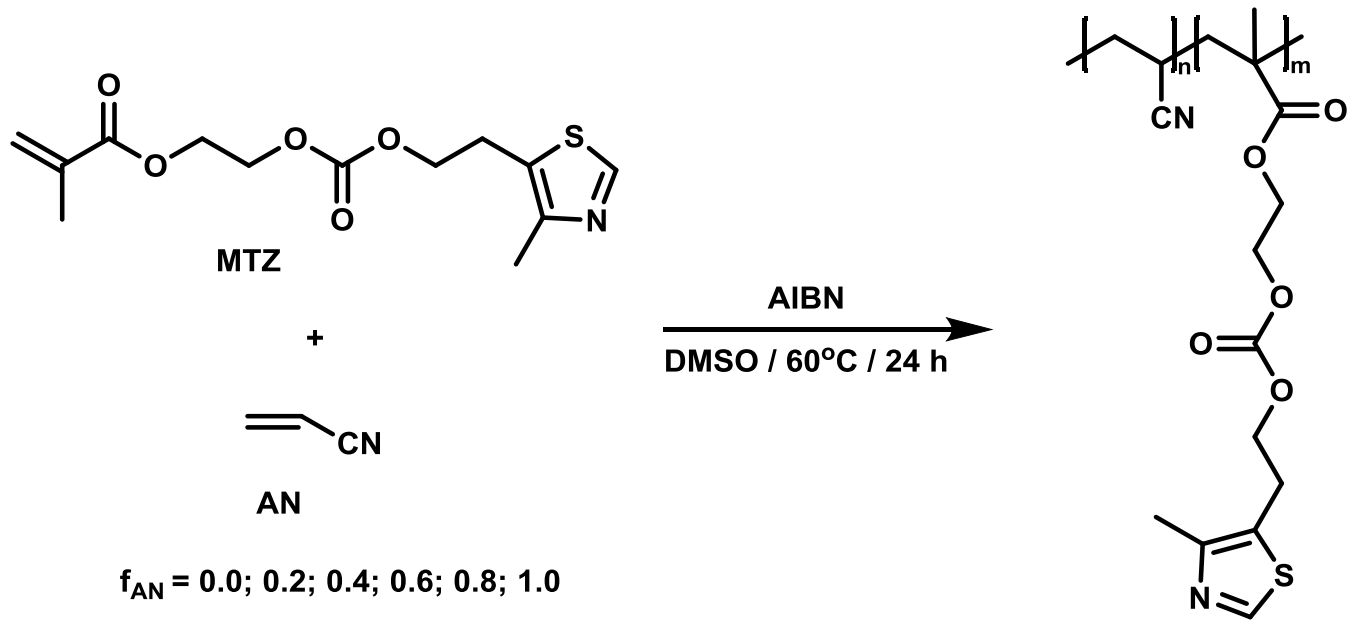

$$
f_{A N}=0.0 ; 0.2 ; 0.4 ; 0.6 ; 0.8 ; 1.0
$$

$\mathbf{P}(\mathrm{AN}-$ co-MTZ)

Scheme 2. Copolymerization reaction of $M T Z$ and $A N$, preparation of $P(A N-c o-M T Z)$ copolymers.

\subsubsection{Quaternization reactions. Synthesis of cationic $P(A N-c o-M T Z-R I)$ copolymers}

Quaternized polymers were obtained using sealed tubes containing magnetic stirring bars. The tubes were charged with the corresponding polymer and a large excess (ratio copolymer/alkyl iodide $1: 3.5)$ of iodomethane or 1-iodobutane in anhydrous DMF (see Scheme 3). Mixtures were purged with argon for $15 \mathrm{~min}$ and heated at $70^{\circ} \mathrm{C}$. Aliquots were taken from the reaction mixture and the degree of quaternization was determined by NMR. Once complete modification was achieved the mixture was cooled down. Samples were first precipitated in hexane under vigorous stirring and then purified by dialysis against distilled water and finally freeze-dried under vacuum.

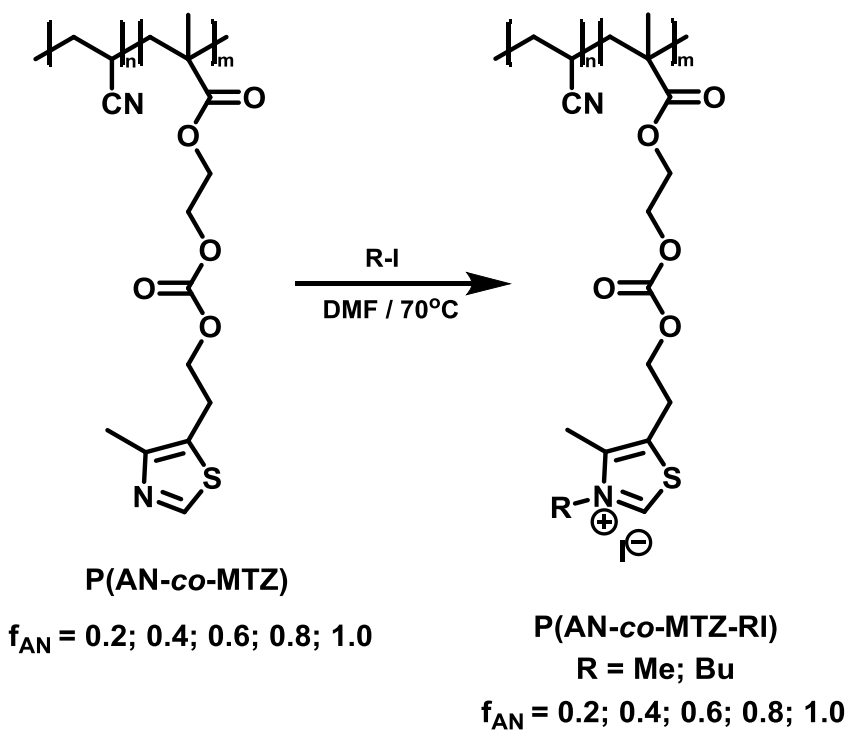

Scheme 3. Quaternization reaction of $\mathrm{P}(\mathrm{AN}-\mathrm{co}-\mathrm{MTZ})$ copolymers synthetized with different acrylonitrile molar fractions $\mathrm{f}_{\mathrm{AN}}$.

\subsubsection{Blends of polymers with polyacrylonitrile, antimicrobial films}

Blends of copolymers P(AN-co-MTZ) and PMTZ with commercial PAN were performed with the purpose of obtaining contact-killing polymeric surfaces. The composition of copolymers or homopolymer was $30 \mathrm{wt} \%$ with respect to PAN, which serves as matrix. The films were obtained by solvent casting from DMF solutions at $10 \% \mathrm{w} / \mathrm{v}$. Each solution was spread in round glass coverslips 
where the solvent was first eliminated at room temperature, followed by a heating treatment at $50^{\circ} \mathrm{C}$ for $18 \mathrm{~h}$ in an oven and finally dried under vacuum until constant weight.

\subsubsection{Microbiology tests}

The antimicrobial activity of copolymers was tested against the different strains, Gram-positive Staphylococcus aureus, Staphylococcus epidermidis, Gram-negative Escherichia coli, Pseudomonas aeruginosa and Candida parapsilosis yeast, according to the Clinical Laboratory Standards Institute (CLSI) microbroth dilution reference method [21]. Strains were cultured on $5 \%$ sheep blood Columbia agar for $24 \mathrm{~h}$ or $48 \mathrm{~h}$ (bacteria or fungi, respectively) at $37^{\circ} \mathrm{C}$, after which a microorganism suspension of $\sim 2 \times 10^{8}$ colony-forming units (CFU) $/ \mathrm{mL}$ in sterile $0.9 \%$ saline was prepared to obtain a turbidity equivalent to that of the $0.5-1.0 \mathrm{McF}$ arland opacity standard and was always used fresh. The inoculum suspension was further diluted 1:100 in Mueller-Hinton broth to yield a microorganism suspension of $\sim 2 \times 10^{6} \mathrm{CFU} / \mathrm{mL}$. Then, each polymer was dissolved in a mixture of sterile water and the minimum amount of DMSO (less than $5 \%, \mathrm{v} / \mathrm{v}$, which was demonstrated to be non-toxic for bacteria) to make a stock solution of $256 \mu \mathrm{g} / \mathrm{mL}$ in which no precipitate appeared. Then, $50 \mu \mathrm{L}$ of broth were added into all the wells of a sterile 96 -well microplate (except in the first column) and $100 \mu \mathrm{L}$ of polymer stock solution was pipetted into the first column of wells. Then, $50 \mu \mathrm{L}$ of polymer solution was diluted by 2-fold serial dilutions in the rest of wells (except in the last column, which will be used as positive control). Briefly, all wells of the broth microdilution plates were inoculated with $50 \mu \mathrm{L}$ of each test microorganism suspensions for a total volume of $100 \mu \mathrm{L}$ to yield the standard density of $5 \times 10^{5} \mathrm{CFU} / \mathrm{mL}$ and affording final polymer concentrations of $128,64,32,16,8,4$, $2,1,0.5,0.25$ and $0.125 \mu \mathrm{g} / \mathrm{mL}$. The plates were then incubated at $37^{\circ} \mathrm{C}$ for $24 \mathrm{~h}$ for bacteria and 48 $\mathrm{h}$ for yeast, respectively. Plates were read for visual turbidity, and minimum inhibitory concentration (MIC) was defined as the lowest polymer concentration at which visible growth of the microorganism was inhibited. All antimicrobial tests were carried out in triplicate and were also repeated on at least two different days. MICs are reported as the most repeated value with an estimated error of \pm one order of dilution.

Antimicrobial activities of the films obtained from blends with PAN were determined against Gram-negative E. coli bacteria by optical density method. Each film was placed in a sterile falcon tube with $1 \mathrm{~mL}$ of broth solution and subsequently $1 \mathrm{~mL}$ of the bacterial suspension was added, resulting $\sim 10^{6} \mathrm{CFU} / \mathrm{mL}$. Blank experiments with glass substrates and with only inoculum were also performed. The tubes were shaken at $120 \mathrm{rpm}$ for $24 \mathrm{~h}$ at $37^{\circ} \mathrm{C}$, and then $200 \mu \mathrm{L}$ of each solution were placed in a 96-well plate. Bacterial growth was determined by the absorption of optical density (OD) at $550 \mathrm{~nm}$ via a microplate reader (VirClia ${ }^{\circledR}$ Chemiluminescence). The measurements were performed at least in triplicate and the antibacterial ratio was estimated as previously described accordingly to the following equation [22]:

$$
\text { Antibacterial ratio }=\frac{O D_{\text {control }}-O D_{\text {Experimental sample }}}{O D_{\text {control }}} \times 100
$$

\section{Results and Discussion}

A novel methacrylic monomer bearing thiazole and carbonate groups, MTZ, was synthesized for the first time with good yield by coupling reaction between HEMAN, obtained by activation of HEMA monomer with $p$-nitrophenyl chloroformate [20], and the commercially available 5-(2-hydroxyethyl)-4-methylthiazole in presence of DMAP and TEA. The successive purifications by column chromatography on silica gel afforded the monomer with high purity as oil, which was characterized by NMR spectroscopy, FTIR and mass spectroscopy. In Figure 1 the proton spectra of HEMA, HEMAN and MTZ monomers are shown. It is easily observed the characteristic peaks of all monomers. The ${ }^{13} \mathrm{C}-\mathrm{NMR}, \mathrm{ATR}-\mathrm{FTIR}$ and MS spectra are shown in the Supporting Information. 


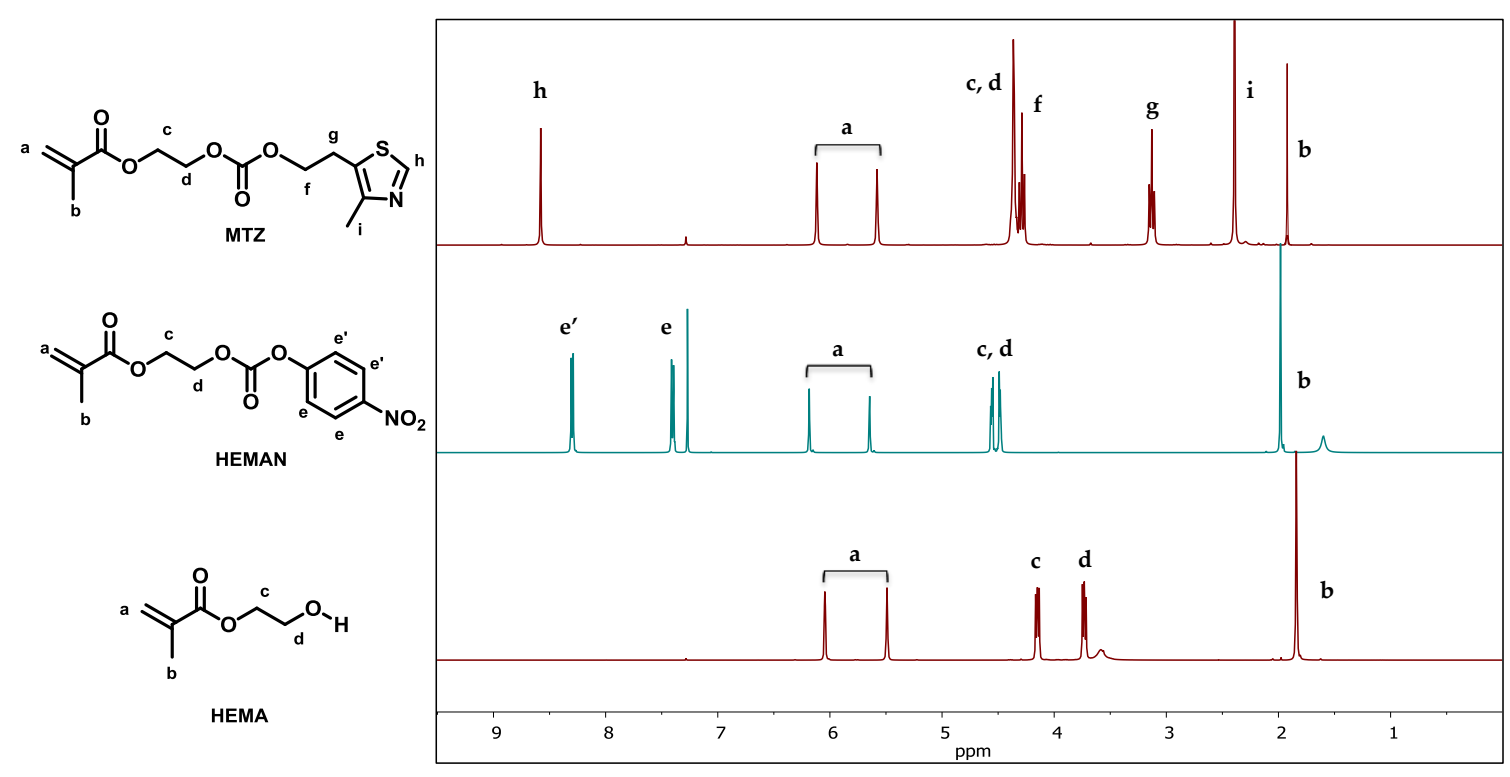

Figure 1. ${ }^{1} \mathrm{H}-\mathrm{NMR}$ spectra of monomers and their corresponding structures.

After the successful synthesis of the MTZ monomer, a series of statistical copolymers of AN and MTZ, P(AN-co-MTZ), were prepared by conventional radical copolymerization in anhydrous DMSO solution at $60{ }^{\circ} \mathrm{C}$ using $5 \times 10^{-2} \mathrm{M}$ of AIBN as initiator concentration and $1 \mathrm{M}$ of monomers total concentration using different $A N$ molar fractions in the feed, $f_{A N}$, ranging from 0.2 to 0.8 . The homopolymers, PMTZ and PAN, were also synthesized in the same reaction conditions (Figures S4 and S5 show the corresponding ${ }^{1} \mathrm{H}$ and ${ }^{13} \mathrm{C}-\mathrm{NMR}$ spectra of the PMTZ homopolymer). All the reactions were carried out during $24 \mathrm{~h}$ to achieve total conversion. Therefore, the final copolymer composition, $F_{A N}$, is practically the same than the molar fraction in the feed. Figure 2 shows the proton NMR spectra of all series. The compositions were calculated by the integral relationship between the thiazole proton at ca. $8.7 \mathrm{ppm}$ and the two methylene protons of AN plus the two methylene protons of the main chain in the range of $1.5-2.2 \mathrm{ppm}$. These copolymer compositions are collected in Table 1 and, as assumed, are likely the initial compositions introduced in the feed.

The number average molecular weights as well as polydispersity indexes were obtained by SEC and the values are also collected in Table 1. SEC curves were also displayed in Figure S6 of Supporting information. As expected by the difference of molar mass between monomers, the $M_{n}$ decreases as the molar fraction of $\mathrm{AN}$ in the copolymer increases until a certain composition from which the $M_{n}$ is almost constant (see Figure S7). The PDI values ranging between 2.1 and 2.5 are consistent with this type of polymerization reaction. 


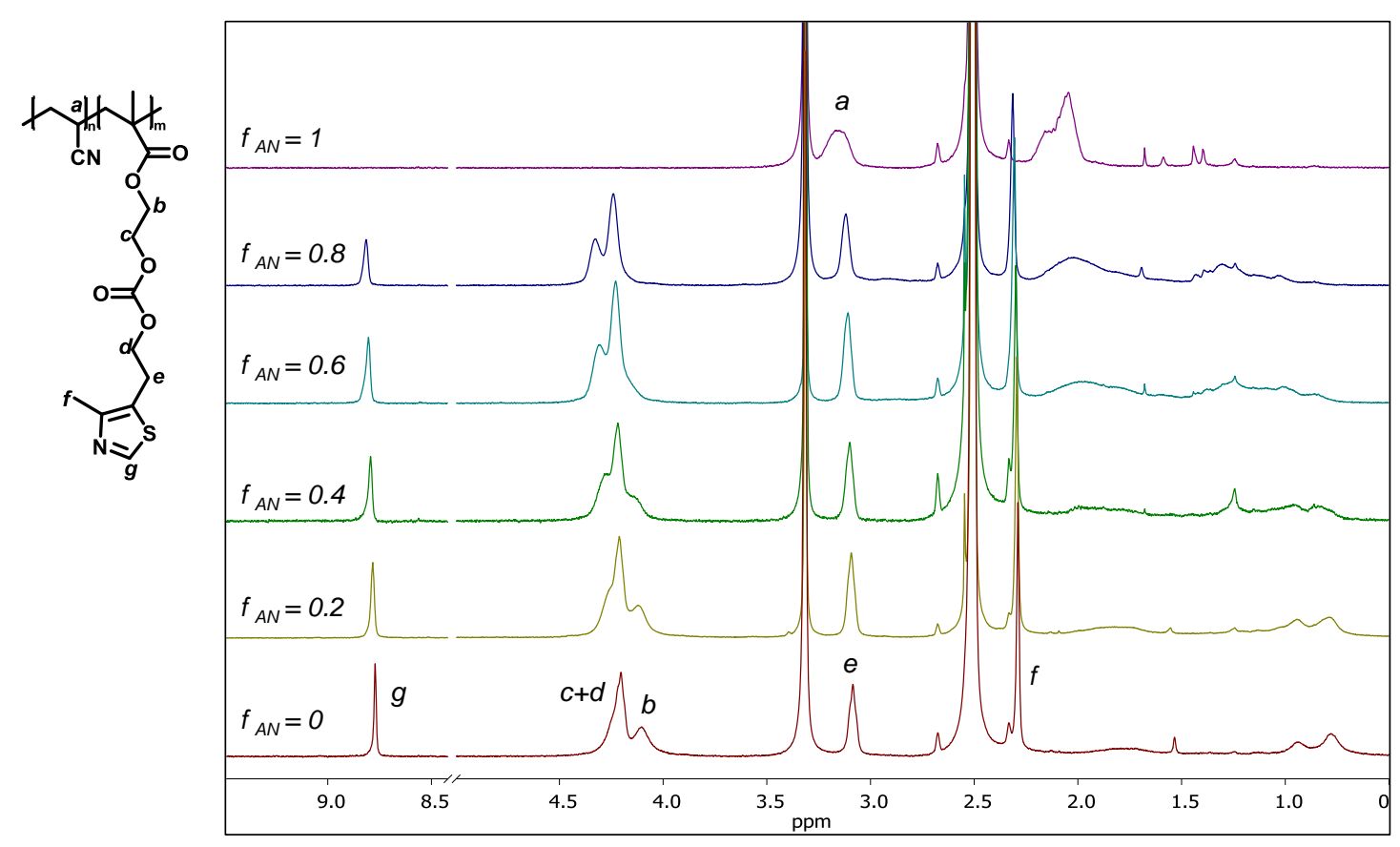

Figure 2. ${ }^{1} \mathrm{H}-\mathrm{NMR}$ spectra of $\mathrm{P}(\mathrm{AN}-\mathrm{co}-\mathrm{MTZ})$ copolymers.

Table 1. Structural parameters of $\mathrm{P}(\mathrm{AN}-\mathrm{co}-\mathrm{MTZ})$ copolymers.

\begin{tabular}{cccc}
\hline $\mathbf{f}_{\mathrm{AN}}$ & $\mathbf{F}_{\mathrm{AN}}$ & $\begin{array}{c}\mathbf{M}_{\mathbf{n}} \\
(\mathbf{D a})\end{array}$ & PDI \\
\hline 0.00 & 0.00 & 78300 & 2.2 \\
0.20 & 0.23 & 43915 & 2.1 \\
0.40 & 0.38 & 23270 & 2.2 \\
0.60 & 0.57 & 19760 & 2.2 \\
0.80 & 0.83 & 17960 & 2.1 \\
1.00 & 1.00 & 17920 & 2.5 \\
\hline
\end{tabular}

Following, the quaternization reactions with alkyl iodides were performed to obtain the corresponding cationic copolymers $\mathrm{P}(\mathrm{AN}-\mathrm{co}-\mathrm{MTZ}-\mathrm{RI})$ with potential antimicrobial activity. The copolymers were quaternized with either methyl or 1-butyl iodide to study the effect of alkyl chain length on the thermal and antimicrobial properties of the copolymers. As an illustrative example, the proton NMR spectra of unquaternized and quaternized homopolymers are displayed in Figure 3 , in the characteristic region of thiazole and thiazolium protons (10.5-8.7ppm). In this region, it is clearly seen the complete alkylation reaction as no remaining thiazole proton (ca. $8.7 \mathrm{ppm}$ ) appears in the corresponding spectra of the quaternized polymers, PMTZ-Bul and PMTZ-Mel. Besides, new bands corresponding to the thiazolium rings formed by alkylation are observed at higher $\delta$ shifts. Similar spectra are perceived for all quaternized copolymers. 


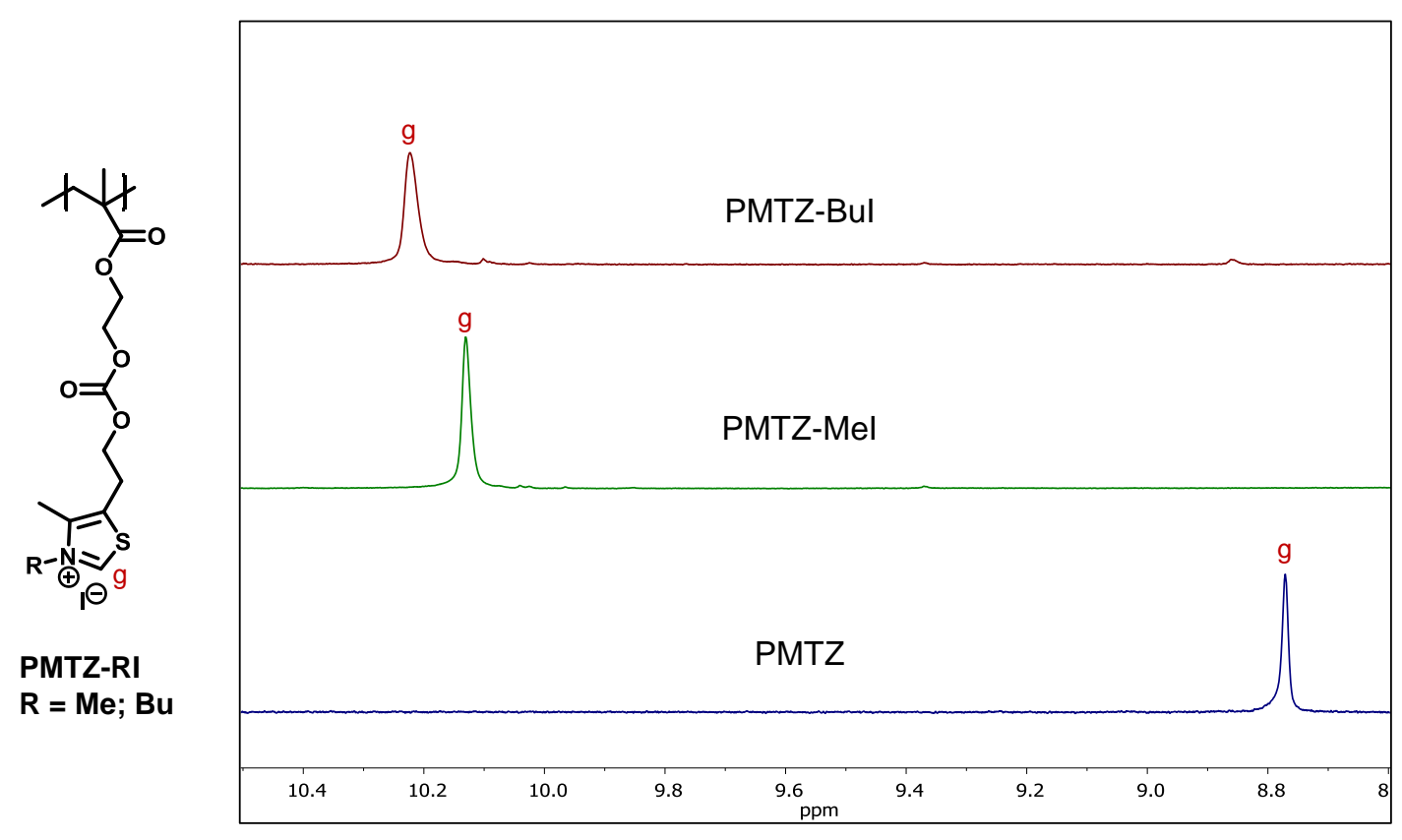

Figure 3. ${ }^{1} \mathrm{H}-\mathrm{NMR}$ spectra characteristic region of thiazole and thiazolium proton of PMTZ and PMTZ-RI homopolymers.

Then, the zeta potential $(\zeta)$ values of modified cationic copolymers were determined in water at room temperature. The obtained values are gathered in Table 2, where is clearly seen that all the copolymers present positive values as a result of the quaternization reaction. Remarkably, it is appreciated that the copolymers quaternized with butyl iodide exhibit higher values than those with methyl iodide, fact that indicates a better stability of amphiphilic copolymers and, in principle, better accessibility of the cationic moieties, which will be reflected in the antimicrobial properties. In addition, in the case of the $\mathrm{P}(\mathrm{AN}-\mathrm{co}-\mathrm{MTZ}-\mathrm{Mel})$ copolymers there is a slender decrease on $\zeta$ values up to intermediate compositions from which start slightly to increase.

Once both series of cationic copolymers, quaternized either with butyl or methyl iodide, were successfully synthesized, an exhaustive study of their thermal properties as a function of the copolymer composition (AN/MTZ ratio), and of the alkylating agents was subsequently performed. In addition, the non quaternized copolymers were also analyzed for comparative purposes. These studies are of great interest for the applicability of these copolymers as antimicrobial materials, for instance as coatings, medical devices or wound dressings.

Firstly, the DSC thermograms of $\mathrm{P}(\mathrm{AN}-\mathrm{co}-\mathrm{MTZ})$ copolymers before quaternization are displayed in Figure $4 a$, in which is observed the glass transition temperature $\left(T_{g}\right)$ of the copolymers (values summarized in Table 3 ). In Figure $4 \mathrm{~b}$ is plotted the variation of $\mathrm{T}_{\mathrm{g}}$ versus MTZ copolymer composition of all copolymers. It is clearly observed in $\mathrm{P}(\mathrm{AN}-\mathrm{co}-\mathrm{MTZ})$ copolymers that $\mathrm{T}_{\mathrm{g}}$ decreases with the incorporation of MTZ in the macromolecular chain up to intermediate compositions from where the $\mathrm{T}_{\mathrm{g}}$ remains constant or slightly increases. The value obtained for $T_{g}$ of PAN is $98.5{ }^{\circ} \mathrm{C}$, which is consistent with data reported in the literature with similar molecular weight [23]. In the case of PMTZ, the lateral chain imparts flexibility and then, its $T_{g}$ reached values below room temperature, $16.0^{\circ} \mathrm{C}$. As happened with other methacrylic polymers bearing thiazole groups previously described by our 
group [24], it is noticeable that the spacer group between the heterocycle pendant groups and the main chain influences the thermal properties.

Table 2. Zeta potential values of $\mathrm{P}(\mathrm{AN}-\mathrm{co}-\mathrm{MTZ}-\mathrm{Mel})$ and $\mathrm{P}(\mathrm{AN}-\mathrm{co}-\mathrm{MTZ}-\mathrm{Bul})$ copolymers in water solution at $25^{\circ} \mathrm{C}$.

\begin{tabular}{ccc}
\hline & \multicolumn{1}{c}{$\zeta(\mathrm{mV})$} \\
\cline { 2 - 3 } $\mathfrak{f}_{\mathrm{AN}}$ & $\mathrm{P}(\mathrm{AN}-$-co-MTZ-Mel $)$ & $\mathrm{P}(\mathrm{AN}-$-co-MTZ-Bul $)$ \\
\hline 0.00 & $41 \pm 5$ & $67 \pm 3$ \\
0.20 & $43 \pm 3$ & $64 \pm 4$ \\
0.40 & $28 \pm 3$ & $61 \pm 1$ \\
0.60 & $40 \pm 1$ & $53 \pm 1$ \\
0.80 & $47 \pm 2$ & $58 \pm 1$ \\
\hline
\end{tabular}
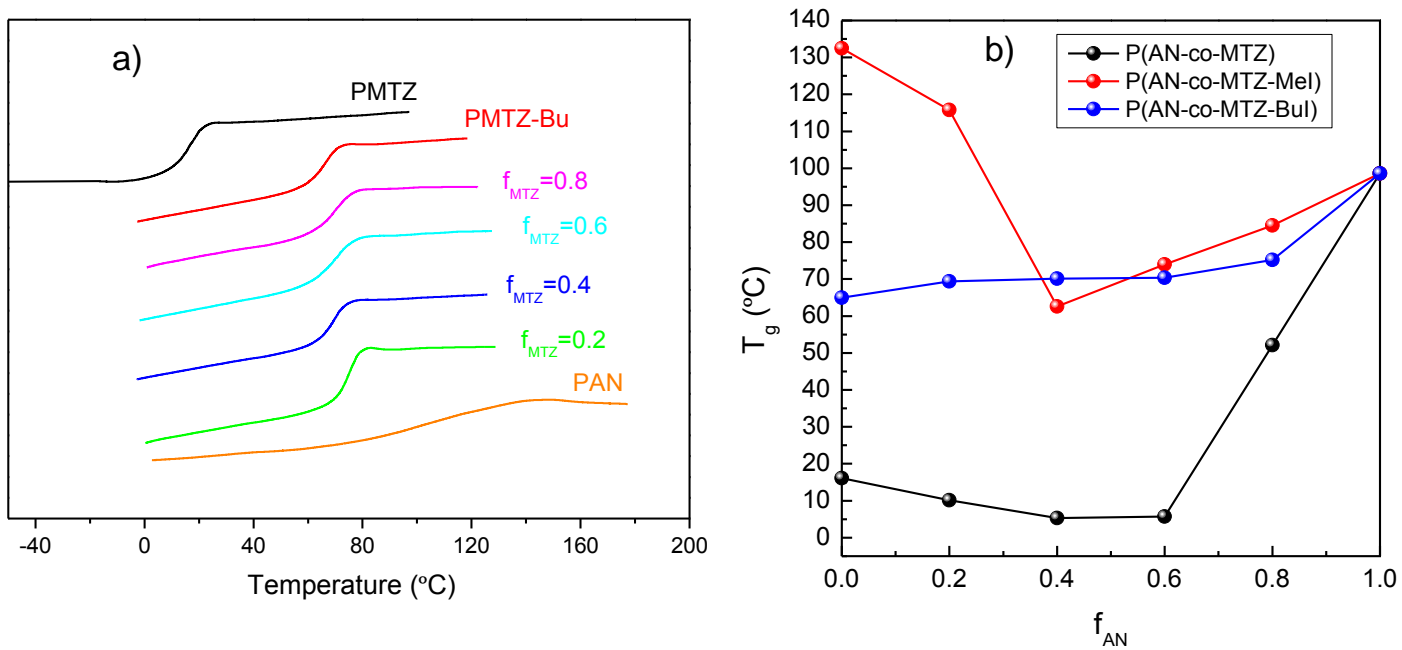

Figure 4. a) DSC Thermograms of $P(A N-c o-M T Z)$ system, and b) glass transition temperature $\left(T_{g}\right)$ variation versus AN composition of all copolymers, $\mathrm{P}(\mathrm{AN}-$ co-MTZ), $\mathrm{P}(\mathrm{AN}-\mathrm{co}-\mathrm{MTZ})$-Mel, $\mathrm{P}(\mathrm{AN}-\mathrm{CO}-\mathrm{MTZ})$-Bul.

Figure $4 \mathrm{~b}$ and Table 3 also display the evolution of the $T_{g}$ with the copolymer composition for the series of the synthesized cationic copolymers, PMTZ-Mel and PMTZ-Bul. It is worthy to remark that both series of cationic copolymers exhibit higher $T_{g}$ values in comparison with the corresponding $\mathrm{P}(\mathrm{AN}-\mathrm{co}-\mathrm{MTZ})$ copolymers. Besides, it is clearly observed that the quaternization reaction with methyl iodide significantly increases the $T_{g}$ of copolymers as previously observed in other studies [25], reaching the homopolymer PMTZ-Mel the highest value with an increment larger than $110^{\circ} \mathrm{C}$. On the other hand, longer alkyl chain length, i.e. butyl, gives more flexibility to the system. Therefore, the $\mathrm{P}(\mathrm{AN}-\mathrm{co}-\mathrm{MTZ}$-Bul) copolymers present much lower glass transition temperatures than $\mathrm{P}(\mathrm{AN}-\mathrm{co}-\mathrm{MTZ}-\mathrm{Mel})$ copolymers. The introduction of carbonate group as spacer gives similar characteristics to previously described homopolymer bearing succinate group [24, 26]. Therefore, it has been demonstrated that the thermal properties of the cationic copolymers can be modulated by varying the composition and the alkylating agent. 
Table 3. Glass transition temperatures $\left(T_{g}\right)$ of the $P(A N-c o-M T Z), P(A N-c o-M T Z-M e l)$ and P(AN-co-MTZ-Bul) copolymers.

\begin{tabular}{cccc}
\hline \multirow{2}{*}{$\mathbf{f}_{\mathrm{AN}}$} & \multicolumn{3}{c}{$\left.\mathbf{T}_{\mathbf{g}} \mathbf{}^{\circ} \mathbf{C}\right)$} \\
\cline { 2 - 4 } & $\mathrm{P}(\mathrm{AN}-$ co-MTZ $)$ & $\mathrm{P}(\mathrm{AN}-$ co-MTZ-Mel $)$ & $\mathrm{P}(\mathrm{AN}-$-co-MTZ-Bul $)$ \\
\hline 0.00 & 16.0 & 132.5 & 65.0 \\
0.20 & 10.0 & 116.0 & 69.5 \\
0.40 & 5.5 & 62.5 & 70.0 \\
0.60 & 6.0 & 74.0 & 70.5 \\
0.80 & 52.0 & 84.5 & 75.0 \\
1.00 & 98.5 & 98.5 & 98.5 \\
\hline
\end{tabular}

Next, the antimicrobial properties in solution of the cationic copolymers were investigated. The activity was tested against two Gram-positive (S. aureus and S. epidermidis) and two Gram-negative ( $P$. aeruginosa and $E$. coli) bacteria and one yeast ( $C$. parapsilosis), all responsible of undesirable hospital-related infection diseases. The MIC values of quaternized copolymers are grouped in Table 4. It is noticeable that $\mathrm{P}(\mathrm{AN}-\mathrm{co}-\mathrm{MTZ}-\mathrm{Mel})$ copolymers are significantly less active than $\mathrm{P}$ (AN-co-MTZ-Bul) copolymers probably due to their lower positive charge density as demonstrated by zeta potential measurements. Besides, it is well known that the hydrophobic/hydrophilic balance of antimicrobial polymers is a key factor influencing the activity [27], therefore the incorporation of long length alkyl chain, i. e. butyl, increases the hydrophobicity, which favors the interaction with the inner hydrophobic part of the membrane and enhances the antimicrobial activity. In fact, the P(MTZ-Mel) homopolymer presents the highest MIC values against most of the tested microorganisms probably due to its higher hydrophilicity. Therefore, when hydrophobic AN is introduced in the macromolecular chain, the effectiveness is improved but when this hydrophobic segment is predominant then, the activity is getting inferior again, as expected. Contrary effect is found for P(MTZ-Bul), the incorporation of butyl chains infers enough hydrophobicity to the polymer and therefore, the best performance is found in this homopolymer. In the corresponding P(AN-co-MTZ-Bul) copolymers, increasing content of $\mathrm{AN}$ diminishes the activity as the incorporation of $\mathrm{AN}$ in the polymer provokes an unbalance between hydrophilic and hydrophobic segments towards hydrophobicity. Moreover and as is habitual observed in cationic antimicrobial systems, the activity is stronger against Gram-positive than against Gram-negative strains, which are composed of double membrane. In the case of fungi, the activity of both copolymeric systems is significantly strong independently of composition. Even when the MTZ composition is as low as 0.2 , there is excellent action.

From the microbiological test results in solution, we confirm that the use of amphiphilic structures, i.e. copolymers with moderate acrylonitrile hydrophobic comonomer or those quaternized with butyl iodide, in general augments the antibacterial activity of the systems. Besides, the incorporation of AN monomeric units in the structure leads to cationic polymers, in principle, more compatible with polyacrylonitrile (PAN). This fact opens the possibility to prepare antimicrobial coatings by blending physical process with commercial available PAN, which is a common material in a variety of biomedical uses [28]. 
Table 4. MIC of quaternized polymers against the different tested stains.

\begin{tabular}{|c|c|c|c|c|c|c|}
\hline \multirow{2}{*}{ Copolymers } & \multicolumn{6}{|c|}{ MIC $(\mu \mathrm{g} / \mathrm{mL})$} \\
\hline & $f_{A N}$ & S. aureus & S. epidermidis & P. aeruginosa & E. coli & C. parapsilosis \\
\hline \multirow{5}{*}{ P(AN-co-MTZ-Mel) } & 0.0 & 128 & 128 & 128 & 128 & 4 \\
\hline & 0.2 & 128 & 8 & 64 & 128 & 4 \\
\hline & 0.4 & 128 & 8 & 128 & 32 & 4 \\
\hline & 0.6 & 4 & 16 & 16 & 128 & 4 \\
\hline & 0.8 & 128 & 32 & 64 & 128 & 8 \\
\hline \multirow{5}{*}{ P(AN-co-MTZ-Bul) } & 0.0 & 4 & 4 & 8 & 4 & 4 \\
\hline & 0.2 & 16 & 16 & 8 & 4 & 4 \\
\hline & 0.4 & 32 & 32 & 16 & 8 & 4 \\
\hline & 0.6 & 64 & 32 & 64 & 16 & 4 \\
\hline & 0.8 & 128 & 32 & 64 & 32 & 8 \\
\hline
\end{tabular}

Then, from $\mathrm{P}(\mathrm{AN}-$-co-MTZ-Mel $)$ and $\mathrm{P}(\mathrm{AN}-$ co-MTZ-Bul) cationic copolymers blend coatings were prepared by solvent casting in DMF. The PAN/copolymer ratio was selected to be 70/30 by weight. Next, to assess the bacterial killing efficiency of these coatings, the films were tested by optical density method against Gram-negative E. coli as model bacteria. Figure 5 shows the antibacterial ratio of the different films containing $30 \%$ of the cationic copolymers and $70 \%$ of commercial PAN. The results reveal that intermediate compositions, i.e. $\mathrm{f}_{\mathrm{AN}}$ of 0.4 , exhibit the highest antibacterial ratio up to $90 \%$, indicating that are very effective to inhibit bacterial growth.

These results are in concordance with those obtained in the microbiological test in solution, in which the copolymers with moderate content of hydrophobic AN units presented low MIC values. This further supports the importance of the hydrophobic/hydrophilic balance on the activity of antimicrobial polymers, not only in solution but also when they are tethered onto surfaces [29-31]. Again, it is noticeable that the films containing the butylated copolymers displayed greater behavior when compared to the corresponding methylated copolymers. It is noteworthy, that the film containing the PMTZ-Bul homopolymer exhibits weak activity ( 17\% antibacterial ratio) contrary to what was expected due to the outstanding properties that this polymer exhibits in solution, MIC of $4 \mu \mathrm{g} / \mathrm{mL}$. Similarly, the methylated homopolymer PMTZ-Mel showed insignificant activity as component of blend coatings. We tentatively ascribe this low performance found in homopolymers when they are part of a coating in comparison with the copolymers, to their low compatibility with PAN. This fact could provoke a phase segregation process in the blend coatings. Therefore, we have demonstrated the significance of designing copolymers composed not only of cationic active monomers but also of other hydrophobic units leading to amphiphilic structures, which the capacity of enhancing the antibacterial activity of the systems. 


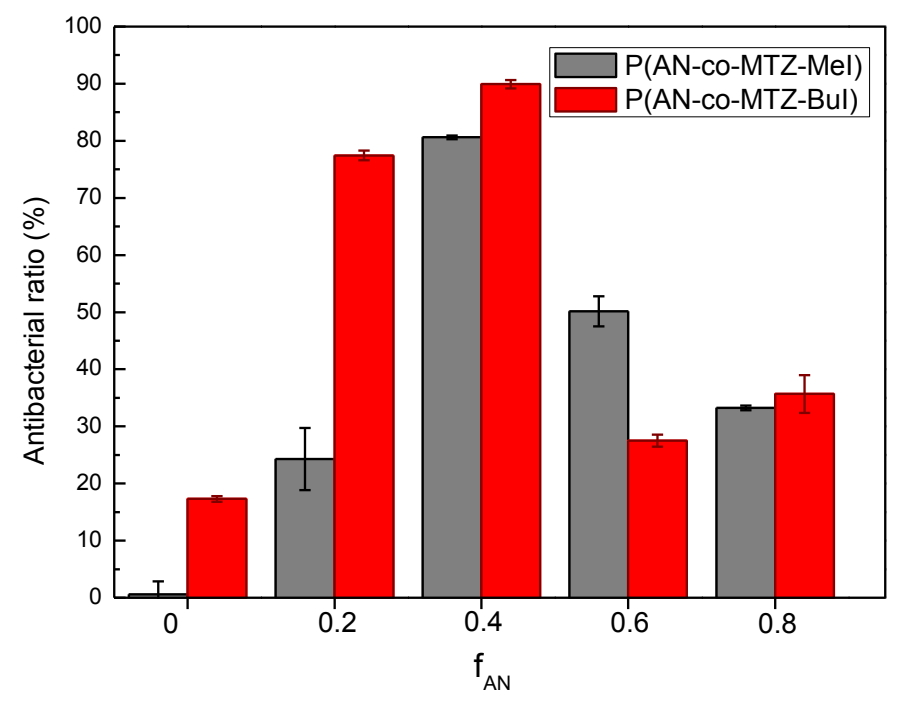

Figure 5. Antibacterial ratio of films obtained from blends composed of commercial PAN and $\mathrm{P}(\mathrm{AN}-\mathrm{co}-\mathrm{MTZ}-\mathrm{RI})$ copolymers.

\section{Conclusions}

This article describes for the first time a novel methacrylic monomer containing thiazole group, in which a hydrolytically sensitive carbonate group is also introduced in the lateral chain acting as extender from the main and lateral chains. This monomer was homo and copolymerized with acrylonitrile by free radical polymerization to obtain polymers with relative high molecular weight. The quaternization with methyl and butyl iodide produces amphiphilic systems with good positive charge and stability, particularly in the case of butyl derivatives. The incorporation of a thiazole group through the formation of carbonate group breaks the strong inter and intramolecular hydroxyl interactions of HEMA homopolymer, giving higher flexibility to PMTZ homopolymer. This is clearly observed in the $T_{g}$ of homopolymer as well as in the copolymers with acrylonitrile. Moreover, the alkylation reaction provokes a notorious change in the thermal behavior of copolymers, resulting the methyl derivatives the most rigid system. Finally, the antimicrobial activity of the copolymers was also evaluated as a function of the alkylating agent and also of the AN content, thus the influence of hydrophilic/hydrophobic balance was investigated. The results revealed that incorporation of hydrophobic AN monomer until intermediate compositions, in general, augments the antibacterial activity of the systems, not only in solution media but also as coating component.

Supporting information: The following are available online: Figure $\mathrm{S} 1:{ }^{13} \mathrm{C}-\mathrm{NMR}$ spectrum of MTZ monomer, Figure S2: FTIR spectrum of MTZ monomer, Figure S3: Mass spectrum of MTZ monomer, Figure S4: ${ }^{1} \mathrm{H}-\mathrm{NMR}$ spectrum of PMTZ, Figure S5: ${ }^{13} \mathrm{C}-\mathrm{NMR}$ spectrum of PMTZ, Figure S6: SEC curves of the synthesized copolymer. Figure S7: Variation of $\mathrm{M}_{\mathrm{n}}$ with the composition in $\mathrm{P}(\mathrm{AN}-\mathrm{co}-\mathrm{MTZ})$ copolymers.

Acknowledgments: This research was funded by MINECO, Project MAT2016-78437-R, the Agencia Estatal de Investigación (AEI, Spain) and Fondo Europeo de Desarrollo Regional (FEDER, EU). C.E. acknowledges the Juan de la Cierva contract (IJCl-2015-26432) from the Spanish Ministry of Science, Innovation. J.A. acknowledges the JAE-Intro program (JAEINT17_EX_0031) from CSIC for his scholarship.

\section{References}

[1] L.J.V. Piddock, The crisis of no new antibiotics—what is the way forward?, The Lancet Infectious Diseases 12(3) (2012) 249-253.

[2] R. Sugden, R. Kelly, S. Davies, Combatting antimicrobial resistance globally, Nature Microbiology 1(10) (2016) 16187. 
[3] J. Guo, J. Qin, Y. Ren, B. Wang, H. Cui, Y. Ding, H. Mao, F. Yan, Antibacterial activity of cationic polymers: side-chain or main-chain type?, Polymer Chemistry 9(37) (2018) 4611-4616.

[4] J. Mayr, J. Bachl, J. Schlossmann, D. Díaz, Antimicrobial and Hemolytic Studies of a Series of Polycations Bearing Quaternary Ammonium Moieties: Structural and Topological Effects, International Journal of Molecular Sciences 18(2) (2017) 303.

[5] Y. Xue, H. Xiao, Y. Zhang, Antimicrobial polymeric materials with quaternary ammonium and phosphonium salts, International Journal of Molecular Science 16(2) (2015) 3626-55.

[6] A. Muñoz-Bonilla, M. Fernández-García, Poly(ionic liquid)s as antimicrobial materials, European Polymer Journal 105 (2018) 135-149.

[7] L. Timofeeva, N. Kleshcheva, Antimicrobial polymers: mechanism of action, factors of activity, and applications, Applied Microbiology and Biotechnology 89(3) (2010) 475-492.

[8] K. Lienkamp, K.N. Kumar, A. Som, K. Nusslein, G.N. Tew, "Doubly selective" antimicrobial polymers: how do they differentiate between bacteria?, Chemistry 15(43) (2009) 11710-4.

[9] K. Matsuzaki, Control of cell selectivity of antimicrobial peptides, Biochim Biophys Acta 1788(8) (2009) 1687-92.

[10] G.N. Tew, D. Liu, B. Chen, R.J. Doerksen, J. Kaplan, P.J. Carroll, M.L. Klein, W.F. DeGrado, De novo design of biomimetic antimicrobial polymers, Proc Natl Acad Sci U S A 99(8) (2002) 5110-4.

[11] R.E. Hancock, H.G. Sahl, Antimicrobial and host-defense peptides as new anti-infective therapeutic strategies, Nat Biotechnol 24(12) (2006) 1551-7.

[12] K. Kuroda, W.F. DeGrado, Amphiphilic polymethacrylate derivatives as antimicrobial agents, Journal of the American Chemical Society 127(12) (2005) 4128-4129.

[13] C. Ergene, K. Yasuhara, E.F. Palermo, Biomimetic antimicrobial polymers: recent advances in molecular design, Polymer Chemistry 9(18) (2018) 2407-2427.

[14] A. Jain, L.S. Duvvuri, S. Farah, N. Beyth, A.J. Domb, W. Khan, Antimicrobial polymers, Adv Healthc Mater 3(12) (2014) 1969-85.

[15] A. Muñoz-Bonilla, M. Fernández-García, Polymeric materials with antimicrobial activity, Progress in Polymer Science 37(2) (2012) 281-339.

[16] R. Tejero, D. López, F. López-Fabal, J.L. Gómez-Garcés, M. Fernández-García, Antimicrobial polymethacrylates based on quaternized 1,3-thiazole and 1,2,3-triazole side-chain groups, Polym. Chem. 6(18) (2015) 3449-3459.

[17] R. Tejero, D. López, F. López-Fabal, J.L. Gómez-Garcés, M. Fernández-García, High Efficiency Antimicrobial Thiazolium and Triazolium Side-Chain Polymethacrylates Obtained by Controlled Alkylation of the Corresponding Azole Derivatives, Biomacromolecules 16(6) (2015) 1844-1854.

[18] R. Tejero, B. Gutiérrez, D. López, F. López-Fabal, J.L. Gómez-Garcés, M. Fernández-García, Copolymers of acrylonitrile with quaternizable thiazole and triazole side-chain methacrylates as potent antimicrobial and hemocompatible systems, Acta Biomaterialia 25 (2015) 86-96.

[19] S. Kasmi, B. Louage, L. Nuhn, G. Verstraete, S. Van Herck, M.J. van Steenbergen, C. Vervaet, W.E. Hennink, B.G. De Geest, Acrylamides with hydrolytically labile carbonate ester side chains as versatile building blocks for well-defined block copolymer micelles via RAFT polymerization, Polymer Chemistry 8(42) (2017) 6544-6557.

[20] O. León, V. Bordegé, A. Muñoz-Bonilla, M. Sánchez-Chaves, M. Fernández-García, Well-controlled amphiphilic block glycopolymers and their molecular recognition with lectins, Journal of Polymer Science Part A: Polymer Chemistry 48(16) (2010) 3623-3631. 
[21] CLSI, Methods for Dilution Antimicrobial Susceptibility Tests for Bacteria That Grow Aerobically; Approved Standard-Ninth Edition, CLSI document M07-A9. Wayne, PA: Clinical and Laboratory Standards Institute2012.

[22] M. Li, X. Liu, Z. Xu, K.W.K. Yeung, S. Wu, Dopamine Modified Organic-Inorganic Hybrid Coating for Antimicrobial and Osteogenesis, ACS Applied Materials \& Interfaces 8(49) (2016) 33972-33981.

[23] M. Minagawa, H. Kanoh, S. Tanno, M. Satoh, Glass-Transition Temperature (Tg) of Free-Radically Prepared Polyacrylonitrile by Inverse Gas Chromatography, 2. Molecular-Weight Dependence of $\mathrm{Tg}$ of Two Different Types of Aqueous Polymers, Macromolecular Chemistry and Physics 203(17) (2002) 2481-2487.

[24] R. Tejero, D. López, M. Fernández-García, Influence of spacer group on the structure and thermal properties of copolymers based on acrylonitrile and methacrylic 1,3-thiazole and 1,2,3-triazole derivatives, European Polymer Journal 71 (2015) 401-411.

[25] A. Muñoz-Bonilla, D. López, M. Fernández-García, Providing Antibacterial Activity to Poly(2-Hydroxy Ethyl Methacrylate) by Copolymerization with a Methacrylic Thiazolium Derivative, International journal of molecular sciences 19(12) (2018) 4120.

[26] R. Tejero, D. López, F. López-Fabal, J.L. Gómez-Garcés, M. Fernández-García, Antimicrobial polymethacrylates based on quaternized 1,3-thiazole and 1,2,3-triazole side-chain groups, Polym. Chem. 6(18) (2015) 3449-3459.

[27] E.F. Palermo, I. Sovadinova, K. Kuroda, Structural determinants of antimicrobial activity and biocompatibility in membrane-disrupting methacrylamide random copolymers, Biomacromolecules 10(11) (2009) 3098-107.

[28] Z.-G. Wang, L.-S. Wan, Z.-K. Xu, Surface engineerings of polyacrylonitrile-based asymmetric membranes towards biomedical applications: An overview, Journal of Membrane Science 304(1) (2007) 8-23.

[29] R. Tejero, B. Gutiérrez, D. López, F. López-Fabal, J. Gómez-Garcés, A. Muñoz-Bonilla, M. Fernández-García, Tailoring Macromolecular Structure of Cationic Polymers towards Efficient Contact Active Antimicrobial Surfaces, Polymers 10(3) (2018) 241.

[30] A. Munoz-Bonilla, R. Cuervo-Rodriguez, F. Lopez-Fabal, J.L. Gomez-Garces, M. Fernandez-Garcia, Antimicrobial Porous Surfaces Prepared by Breath Figures Approach, Materials (Basel) 11(8) (2018) 1266-1276.

[31] M. Alvarez-Paino, P. Bonilla, R. Cuervo-Rodríguez, F. López-Fabal, J.L. Gómez-Garcés, A. Muñoz-Bonilla, M. Fernández-García, Antimicrobial surfaces obtained from blends of block copolymers synthesized by simultaneous ATRP and click chemistry reactions, European Polymer Journal 93 (2017) 53-62. 